\title{
Investigation of quantum transport and local density of states in a graphene strip connected to a square lattice
}

\author{
Elnaz Rostampour ( $\sim$ el_rostampour@yahoo.com ) \\ Urmia University https://orcid.org/0000-0002-5318-1138
}

\section{Research Article}

Keywords: graphene quantum billiard, Quantum Transport, the local density of state

Posted Date: February 14th, 2022

DOI: https://doi.org/10.21203/rs.3.rs-371781/v2

License: (c) (1) This work is licensed under a Creative Commons Attribution 4.0 International License.

Read Full License 


\title{
Investigation of quantum transport and local density of states in a graphene strip connected to a square lattice
}

\author{
Elnaz Rostampour \\ Department of Physics, Urmia University, 11 k.m Serow Road, Urmia, Iran \\ el_rostampour@yahoo.com
}

\begin{abstract}
We theoretically express quantum transport at Dirac points via graphene quantum billiard as a non-magnetic material to connect metallic leads. Our results indicate that the quantum billiard of graphene is similar to a resonant tunneling device. The centerpiece size and the Fermi energy of the graphene quantum billiard play an important role in resonant tunneling. In graphene, a change of carrier density can affect plasmon polaritons. At the Dirac point, the conductivity of graphene depends on the geometry, so that the conduction of the evanescent modes is close to the theoretical value of $4 \mathrm{e}^{2} / \pi \mathrm{h}$ (where Planck's constant and the electron charge are denoted by $\mathrm{h}$ and e, respectively.). This transport property can be used to justify chaotic quantum systems and ballistic transistors. Our theoretical results demonstrate that the local density of state of the graphene sheet for $\epsilon_{L}=\epsilon_{R}=0$ is larger than $\epsilon_{L}=\epsilon_{R}=t$ (where $\epsilon_{L}\left(\epsilon_{R}\right)$ is onsite energy of the left (right) metallic lead) unlike the current obtained from the calculations.
\end{abstract}

Keywords: graphene quantum billiard, Quantum Transport, the local density of state

\section{Introduction}

Recently, the tunneling magnetoresistance (TMR) effect of hybrid materials with both organic molecules and ferromagnetic materials has been studied for junctions [1,2] and granular substances [3]. At normal incidence, Klein tunneling in graphene is considered to be completely transparent of barriers, as was done by Katsnelson et al. [4] and experimentally [5], causing undesired charge leakage in graphene devices. Lack of Klein tunneling [6] in Particle Physics unlike graphene, encapsulated electrons seem to be a practical and challenging task. In graphenebased nano-devices, the confinement of carriers is enhanced, such as $p$ - $n$ junction [7], superlattices [8], and FET [9]. To control quantum transport in graphene nanorings, the effects of interference of transport of coherent electrons are considered by ignoring potential barriers. In the case mentioned, research conducted by $\mathrm{Wu}$ et al. [10] has shown that the behavior of the device is similar to resonance tunneling, and the external magnetic field or the size of the device is effective in regulating the resonant energy. By applying the electrostatic potential to an arm on 
the Aharonov-Bohm magnetoconductance, its effects could be examined in [11]. Pel et al.[12] showed that fluctuations in mesoscopic conductivity in graphene depend on the Hamilton symmetry properties, indicating the nontrivial universality of graphene. In disordered monolayer graphene, valley symmetry on UCF has also been studied. There is a linear 2D energy spectrum near the Dirac points, and the moving electrons have a constant speed and the band velocity of $v_{F}=10^{6} \mathrm{~m} / \mathrm{s}$. The dispersion relation in graphene has interesting electronic properties similar to relativistic and massless fermions. In a hexagonal carbon lattice, such as single-walled carbon nanotubes, charge carriers play an important role in electronic transport $[13,14]$. High mobility graphene specimens are used to make mesoscopic graphene devices. In this work, we study quantum transport in graphene quantum billiard with ferromagnetic leads.

\section{Methodology}

A graphene strip with lattice parameter $a$ is considered as in Figure 1, which is connected on both sides by metallic leads to a square lattice with the same lattice parameter [15]. Hamiltonian of this model is usually described as:

$H=H_{C}+\sum_{\alpha=L, R}\left(H_{\alpha}+H_{\alpha C}\right)$

The first term of equation (1) is channel Hamiltonian that described by the tight -binding model:

$H_{C}=\sum_{<i, j>, \sigma} t\left(c_{i, \sigma}^{\dagger} c_{j, \sigma}+H . c.\right)$

We determine the hopping integral at the bond $\langle i, j\rangle$, with $t=t_{0}+t_{0} e^{i k_{y}}$ if $i=$ $1,5,9, \ldots$ and $0<i<N-1, t=t_{0}$ if $i=2,6,10 \ldots$ and $0<i<N-1, t=t_{0}+t_{0} e^{-i k_{y}}$ if $i=3,7,11 \ldots$ and $0<i<N-1$ and $t=t_{0}$ if $i=4,8,12 \ldots$ and $0<i<N-1$. In the above equation, $t_{0}$ is hopping integral between nearest neighbour $i$ th and $j$ th sites of the ideal system, $k_{y}$ is the momentum in the $y$ direction in the range $-\frac{\pi}{a}<k_{y}<\frac{\pi}{a}$ and the sum is on the nearest neighboring atoms $\langle i, j\rangle$. The annihilation operator is denoted by $c_{i, \sigma}$ for electron $\pi$ at the site $i$ with spin $\sigma$ [spin-up $(\uparrow)$ or spin-down $(\downarrow)$ ]. The left (right) metallic lead Hamiltonian is also expressed by the tight binding model. The Hamiltonian of left (L) and right (R) electrodes, $H_{\alpha}$, is described as:

$H_{\alpha}=\sum_{i, \alpha} \epsilon_{i, \alpha} c_{i \alpha, \sigma}^{\dagger} c_{j \alpha, \sigma}$

Here, $\epsilon_{L}\left(\epsilon_{R}\right)$ is onsite energy of left (right) metallic lead, $t_{L}\left(t_{R}\right)$ is nearest neighbor hopping amplitudes of the left (right) metallic lead, $\epsilon_{i, \alpha}$ is $\epsilon_{i, L}=\epsilon_{L}+2 t_{L} \cos k_{y}\left(\epsilon_{i, R}=\epsilon_{R}+2 t_{R} \cos k_{y}\right)$ of left (right) electrode. The coupling matrix is expressed by $H_{\alpha C}$ between surface atomic orbital of leads and channel as:

$H_{\alpha C}=\sum_{\alpha=L, R} \sum_{i \alpha, j \alpha, \sigma}-t_{\alpha}^{\prime}\left(c_{i \alpha, \sigma}^{\dagger} d_{j c, \sigma}+\right.$ h.c. $)$

Where $t_{L}^{\prime}\left(t_{R}^{\prime}\right)$ is hopping amplitude between graphene sample and left (right) lead (for $\mathrm{i}=0$ and $\mathrm{N})$. The spin-up $(\uparrow)$ current plus the spin-down $(\downarrow)$ current is total current [16-19] as:

$I\left(V_{b}, V_{g}\right)=\frac{e}{h} \int_{-\infty}^{+\infty}\left[f\left(\varepsilon-\mu_{L}\right)-f\left(\varepsilon-\mu_{R}\right)\right] T\left(\varepsilon, V_{b}, V_{g}\right) d \varepsilon$ 
where $e, h, f$ and $\mu_{L, R}=E_{f} \pm \frac{1}{2} e V_{b}$ are electron charge, Planck's constant, Fermi-Dirac distribution function, and electrochemical potentials of right and left electrodes, respectively. The dependence of the transmission functions on gate and bias voltages, spin and energy is shown by the relation $T^{\sigma}\left(\varepsilon, V_{b}, V_{g}\right)=\operatorname{tr}\left[\Gamma_{L}^{\sigma} G_{c, \sigma}^{R} \Gamma_{R}^{\sigma} G_{c, \sigma}^{A}\right]$, where $G_{c, \sigma}^{R(A)}, \Gamma_{L(R)}^{\sigma}=i\left(\sum_{L(R), \sigma}-\right.$ $\sum_{L(R), \sigma}^{\dagger}$ ) and $\sum_{L(R), \sigma}$ are retarded (advanced) Green's function with $\sigma$ spin, the injection ratio, and the self-energy with $\sigma$ spin at sites $1(\mathrm{~N})$, respectively,

$\sum_{L(N), \sigma}(\varepsilon)=t_{L(N)}^{\prime} e^{-i k_{L(R), \sigma}}$

with

$k_{L(R), \sigma}=\arccos \left(\frac{\varepsilon-\epsilon_{\mathrm{L}(\mathrm{R})}-2 \mathrm{t}_{\mathrm{L}(\mathrm{R})} \cos k_{\mathrm{y}}}{2 \mathrm{t}_{\mathrm{L}(\mathrm{R})}}\right)$.

Despite the bias voltage, the Green function of the system can be obtained by using the effective Hamiltonian of the molecule $\left(H_{C}\right)$ and self-energy functions $\left(\sum\right)$ :

$G_{C, \sigma}^{R, A}(\varepsilon)=\frac{1}{(\varepsilon \pm i \eta) I+H_{C}-\sum_{L, \sigma}-\sum_{R, \sigma}}$

Where $\eta=0^{+}$is considered a positive infinitesimal constant. Finally, the density of state function is as $\operatorname{DOS}(\varepsilon)=\frac{1}{2 \pi} \operatorname{Tr}(A(\varepsilon))$ where is calculated from the density of state matrix $A(\varepsilon)=i\left(G^{R}-\right.$ $\left.G^{A}\right)$.

\section{Results and discussions}

Based on the two-atom basis, the group velocities of the Bloch ansatz are very high near those points at $\sim 8.5 \times 10^{5} \mathrm{~m} / \mathrm{s}$, indicating an effective " speed of light " in the comparison of massless Dirac fermions. The $\pi$ and $\pi^{*}$ zone-center $\Gamma$ energies symmetrically to $E_{F}$ represent the orthogonal tight-binding model. When the overlap integral is not zero, the antibonding $\pi^{*}$ bands are at higher energies. The parameter $\pi-\pi^{*}$ has similar results (Reich et al., 2002), as well as more accurate calculations of first-principles. The effects beyond nearest-neighbor interactions and the so-called trigonal warping correction can also be examined. Periodic conduction fluctuations with bias and gate voltage are caused by quantum interference of graphene at low temperatures by multiply-reflected waves of electrons and holes. Graphene is considered a quantum billiard with a long phase coherence length of more than $5 \mu \mathrm{m}$ [20]. The experimental concept of Andreev billiard in graphene is the long phase coherence length of charges. Such a device represents the new Josephson junction in which the electron system in normal metal is Dirac fermions, not a Fermi Liquid. These junctions are predicted to exhibit a number of novel phenomena, such as Andreev reflection look like mirror [21, 22], Andreev electrons with a new type of propagating mode in the normal metal channel [23], and fluctuation of transmission probability depending on the width of the barrier [24]. In mesoscopic systems, fluctuations in quantum conductivity within the carrier confinement to a phase space with decreasing dimensions are visible [25-28]. The results obtained for the $\mathrm{I}-\mathrm{V}$ characteristics of graphene are depicted in Figure 2 by the value of $k_{y}$ (adjacent to Dirac point), $\left(\frac{4 \pi}{3 a}\right)$, that the current by increasing the voltage increases. Bias voltages are reasonable in practical experimental measurements in the range from 0.0 to $2 \mathrm{~V}$. The current doesn't drop in graphene attached to 
square lattice leads. For $t_{L}=t_{R}=\epsilon_{L}=\epsilon_{R}=t$ we obtain a larger current than when $t_{L}=t_{R}=$ $t, \epsilon_{L}=\epsilon_{R}=0$. By changing the lead energy band centers, these values change. Recently several groups have performed non-local four-probe measurements [29, 30, 31] in graphene, attached to a ferromagnetic electrode, where there are spin currents between injector and detector. At room temperature, graphene experiments with a spin relaxation length greater than $2 \mu \mathrm{m}$ allow the injection of a spin current [29].

In graphene connected to two ferromagnetic electrodes, magnetic resistance of several hundred Ohms is visible in the rotation valve [32] which is also used in spintronic devices. Leaddetermining parameters affect the conductance, $G=\frac{e^{2}}{h} \frac{2 g_{s} g_{v}}{\pi} \frac{W}{L} \frac{\sin k_{L} \sin k_{R}}{\sin \left(k_{L}+k_{R}\right)} \arctan \left(\frac{\sin \left(k_{L}+k_{R}\right.}{1-\cos \left(k_{L}+k_{R}\right)}\right)$ where $W$ and $L$ are the wide and length of the graphene sample, respectively. The $g_{s(v)}=2$ is the spin (valley) degeneracy. The relations $k_{L}$ and $k_{R}$ show the dependence of conductance only on the parameters of the lead via $t_{L}$ and $t_{R}$. The conductance in terms of the function of $t_{L}$ and $t_{R}$ at the Dirac points, $\left(0, \frac{4 \pi}{3 a}\right)$, is plotted in Figure 3. The probability of transmission in terms of a function of energy with $t_{L}=t_{R}=\epsilon_{L}=\epsilon_{R}=t$ and $t_{L}=t_{R}=t, \epsilon_{L}=\epsilon_{R}=0$ at the Dirac point $\left(\frac{4 \pi}{3 a}\right)$ represents at the bias of $0 \mathrm{~V}$ in fig.4. Near the Dirac points of graphene, the probability of transmission can be examined from the point of view of the excitations of a virtual electronhole pair close to zero energy [33]. Transmission resonances depend on doping and confinement in the quantum transport. To expand the energy level, the effect of self-energy is used, which due to the rule of sum, its integrated strength does not change. The self-energy method allows us to investigate in detail the local density of states (LDOS) in different parts of graphene. LDOS tells us the number of energy Eigen-state per unit energy range and it depends on dispersion relation. The size quantization effects modify it. In Fig.5, LDOS of the graphene at the left end and right end are depicted as a function of $\mathrm{E}(\mathrm{eV})$ that is equal for the two ends. LDOS of the graphene at the Dirac point for $t_{L}=t_{R}=t, \epsilon_{L}=\epsilon_{R}=0$ is larger than $t_{L}=t_{R}=\epsilon_{L}=\epsilon_{R}=t$ (Fig.5(b)). Plasmon propagation distance is checked from experimental data calculated LDOS diagrams in Ref. 34.

Localized graphene plasmon resonance in the LDOS model is generated in localized modes for specific values of bandwidth $(W=0.37 \lambda p$ and $0.82 \lambda p)$ near the graphene ribbon strip. To increase the plasmon-dipole interaction and the signal in the near-field, a strong concentration of electromagnetic field is used [34]. In theory and experiment, the profiles of the two localized modes and the conventional Fabry-Perot cavity modes are both completely different from each other. At the edges of the graphene there is a maximum field in the lowest order mode and in the middle there is a maximum field in the lowest order of conventional Fabry-Prote mode. It should be noted that the reflection coefficient of graphene plasmons at the boundaries is approximately one (zero phase). 


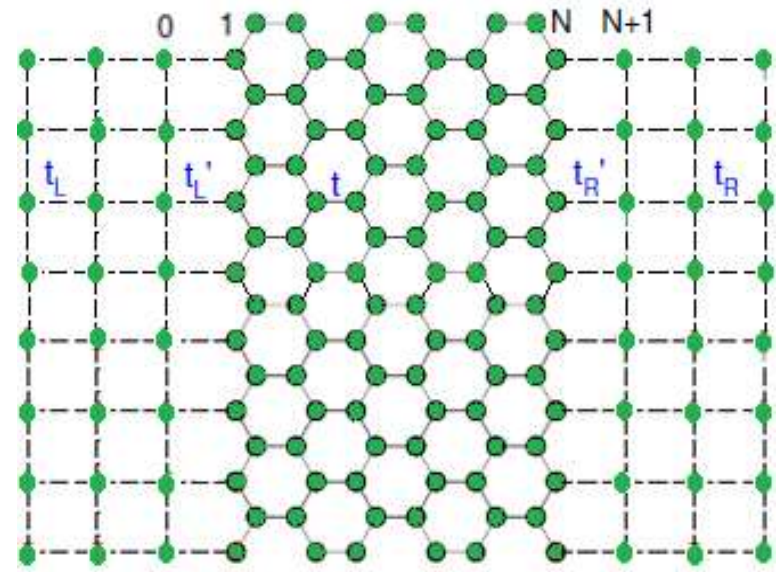

Figure 1. A graphene sample attached to metallic leads and matched square lattics connected to it.

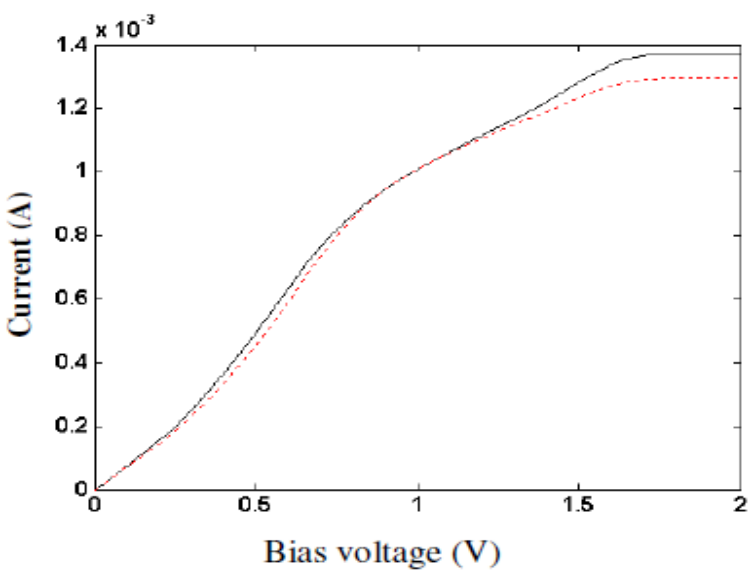

Figure 2. Current versus voltage characteristics for graphene with $t_{L}=t_{R}=\epsilon_{L}=\epsilon_{R}=t$ $\left(\right.$ solid plot (-)) and $t_{L}=t_{R}=t, \epsilon_{L}=\epsilon_{R}=0$ (dashed plot (-)).
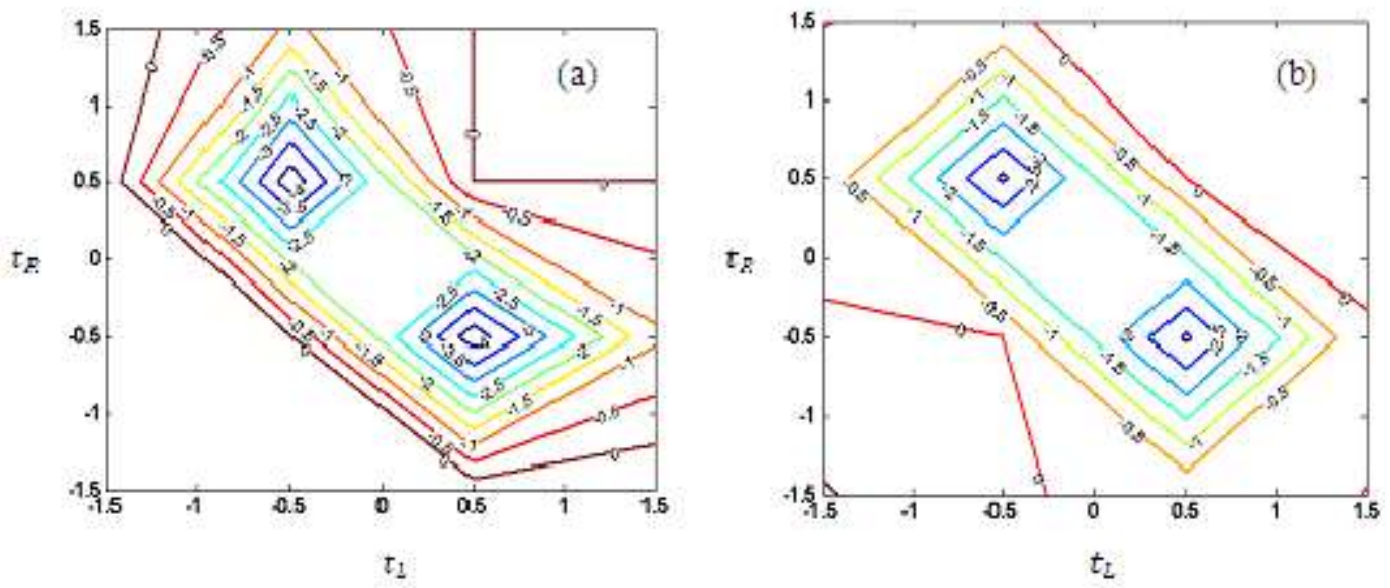
Figure 3. Conductance evaluated at the Dirac points, $\varepsilon=0$, (a) $k_{y}=0$, (b) $k_{y}=\frac{4 \pi}{3 a}$, in terms of the source and drain leads parameters.

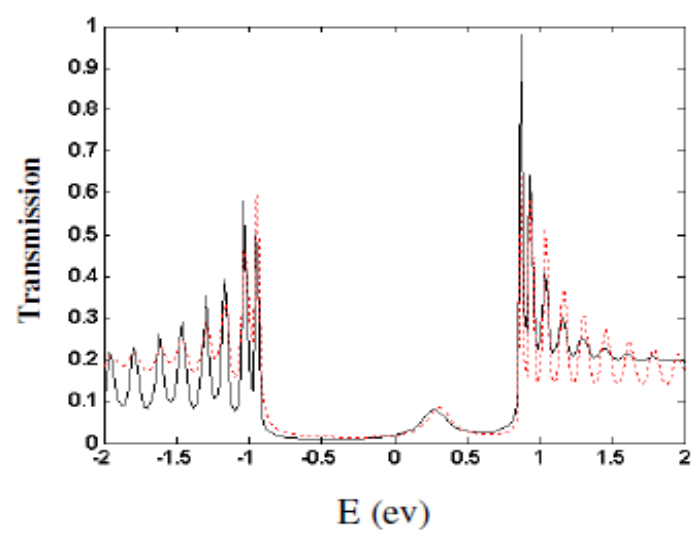

Figure 4. The probability of transmission in terms of energy at $V_{b}=0 \mathrm{~V}$ with $t_{L}=t_{R}=\epsilon_{L}=$ $\epsilon_{R}=t(\operatorname{solid}$ plot $(-))$ and $t_{L}=t_{R}=t, \epsilon_{L}=\epsilon_{R}=0$ (dashed plot (-)).

(a)

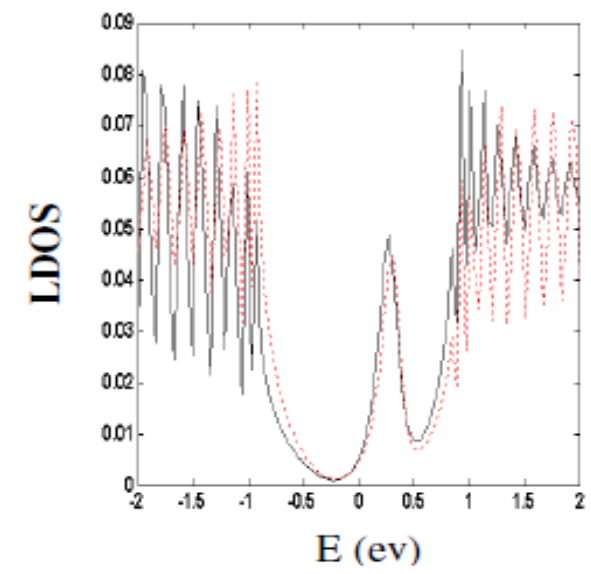

(b)

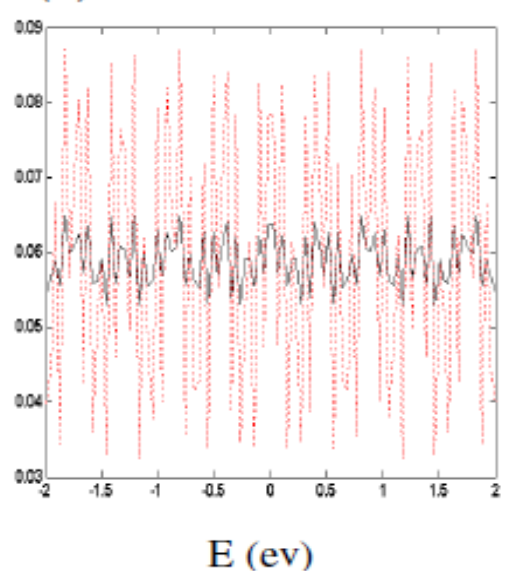

(c)

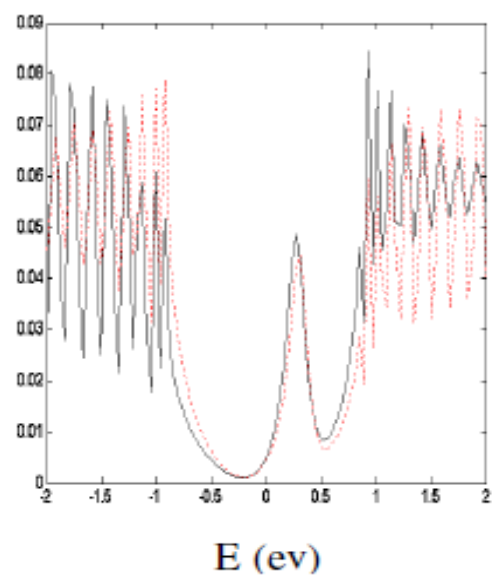

Figure 5. LDOS (a) at left end of graphene, (b) of the graphene for $\mathrm{i}=1, \mathrm{~N}$ and (c) at right end of graphene with $t_{L}=t_{R}=\epsilon_{L}=\epsilon_{R}=t$ (solid plot (-)) and $t_{L}=t_{R}=t, \epsilon_{L}=\epsilon_{R}=0 \quad$ (dashed plot $(-))$.

\section{Conclusions}

The quantum transport is discussed in graphene quantum billiard by the non-equilibrium Green function method. Here we have the increase in current with the increase in voltage. The graphene quantum billiard is suggested for the operation of devices with a nanoscale current. Our theoretical results indicate that the local density of the state is equal in the left and right metallic leads. Furthermore, onsite energies of left and right metallic leads $\left(\epsilon_{L}\right.$ and $\left.\epsilon_{R}\right)$ affect the quantum transport through graphene quantum billiard with ferromagnetic leads.

\section{Conflict of Interest}


The author declares that she has no conflict of interest.

\section{References}

[1] Z.H. Xiong, D. Wu, Z. Valy Vardeny, J. Shi, Nature 427, 821 (2004).

[2] S. Miwa, M. Shiraishi, S. Tanabe, M. Mizuguchi, T. Shinjo, Y. Suzuki, Phys. Rev. B 76, 214414-1(2007).

[3] S. Sakai, I. Sugai, S. Mitani, K. Takanashi, Y. Masumoto, H. Naramoto, P.V. Avramov, S. Okayasu, Y. Maeda, Appl. Phys. Lett. 91 , 242104 (2007).

[4] M. I. Katsnelson, K. S. Novoselov and A. K. Geim, naturephysics,384, 2 ,620-625(2006)

[5] K. S. Novoselov, A. K. Geim, S. V. Morozov, D. Jiang, M. I. Katsnelson, I. V. Grigorieva, S. V. Dubonos and A. A. Firsov, Nature 438, 197-200 (2005)

[6] A. F. Young and P. Kim,naturephysics, 5 ,222-226 (2009)

[7] H.S. Byung , Y. Jongmin , and S. Moonsub, ACS Nano, 8 (9), pp 8831-8836(2014)

[8] A. Reserbat-Plantey, D. Kalita, Z. Han, L. Ferlazzo, S. Autier-Laurent, K. Komatsu, C. Li, R.Weil, A. Ralko, L. Marty, S. Gueron, N. Bendiab, H. Bouchiat, and V. Bouchiat, Nano Lett. 14, 5044-5051 (2014).

[9] S. Kim, J. Nah, I. Jo, D. Shahrjerdi, L.Colombo,Z. Yao, E. Tutuc and S. K. Banerjee, APPLIED PHYSICS LETTERS 94, 062107 (2009).

[10] Z. Wu, Z. Z. Zhang, K.Chang and F. M. Peeters, Nanotechnology 21185201 (2010).

[11] A. Rycerz, ACTA PHYSICA POLONICA A 115,322-325 (2009).

[12] A. N. Pal, V. Kochat and A. Ghosh,e-print arxiv:condmat/1206.3866

[13] T. Ando, T. Nakaishi, R. Saito, J. Phys. Soc. Japan 67, 2857(1998).

[14] P.L. McEuen, M. Bockrath, D.H. Cobden, Y. Yoon, S.G. Louie, Phys. Rev. Lett. 83 5098(1999).

[15] H.Schomerus, cond-mat/0611209.

[16] B D Cullity and C D Graham, Introduction to Magnetic Materials

(Edis) L Hanzo (New York: Wiley-IEEE) p 116 (2008)

[17] Z Yang, C Hu and Q Meng Indian J. Phys. 86977 (2012)

[18] S Datta, Electronic Transport in Mesoscopic Systems,Cambridge

(edis) H Ahmad, M Pepper, A Broers (New York: Cambridge

University Press) p 293 (1995)

[19] Y Xue, S Datta and M A Ratner Chem. Phys. 281151 (2002)

[20] F. Miao, S. Wijeratne, Y. Zhang, U. C. Coskun, W. Bao, C. N. Lau , Science 137 (5814) 1530-1533, 2007

[21] C.W.J. Beenakker, Specular andreev Reflection in graphene. Physical Review Letters, 2006. 97(6): p. 067007.31.

[22] M. Titov, and C.W.J. Beenakker, Josephson effect in ballistic graphene. Physical Review B, 2006. 74(4): p. 041401.

[23] M. Titov, A.O., C. W. J. Beenakker, Excitation gap of a graphene channel with superconducting boundaries. cond-mat/0609623, 2006.

[24] K. Sengupta, , Josephson effect in graphene SBS junctions. cond-mat/0611614.

[25] van Wees, B. J. et al. Observation of zero-dimensional states in aone-dimensional electron interferometer. Phys. Rev. Lett. 62, 2523_2526 (1989).

[26] Y.Ji, et al. An electronic Mach-Zehnder interferometer. Nature 422,415_418 (2003). 
[27] W. Liang, et al. Fabry_Perot interference in a nanotube electron waveguide. Nature 411, 665_669 (2001).

[28] F. Miao, et al. Phase-coherent transport in graphene quantum billiards. Science 317, 1530_1533 (2007).

[29] N. Tombros, C. Jozsa, M. Popinciuc, H. T. Jonkman, and B. J. van Wees, condmat/0706.1948; L. Brey and H. A. Fertig, arXiv:0710.2019v1 [cond-mat.mes-hall] 2007.

[30] S. Cho, Y.-F. Chen, and M. S. Fuhrer, cond-mat/0706.1597.

[31] M. Ohishi, M. Shiraishi, R. Nouchi, T. Nozaki, T. Shinjo,and Y. Suzuki, Jap. Journal of Appl. Phys. 46, L605

(2007).

[32] E.Hill, A.K.Geim, K.Novoselov, F.Schedin, and P.Blake,IEEE Trans. Magn. 42, 2694 (2006).

[33] J. Tworzydlo, B. Trauzettel, M. Titov, A. Rycerz, and C. W. J. Beenakker, Phys.Rev.Lett. 96, 246802 (2006).

[34] F. H. L. Koppens, D.E. Chang, and F.J. Garcia de Abajo, NanoLett!11, 3370-3377(2011).

Declarations

Funding (information that explains whether and by whom the research was supported)

Not applicable

Conflicts of interest/Competing interests (include appropriate disclosures)

Not applicable

Ethics approval (include appropriate approvals or waivers)

Not applicable

Consent to participate (include appropriate consent statements)

Not applicable

Consent for publication (consent statement regarding publishing an individual's data or image)

Not applicable

Availability of data and material (data transparency)

Not applicable

Code availability (software application or custom code)

Not applicable

Authors' contributions (Individual author's contributions)

Not applicable 